\title{
FUSION RULES OF VIRASORO VERTEX OPERATOR ALGEBRAS
}

\author{
XIANZU LIN \\ (Communicated by Kailash C. Misra)
}

\begin{abstract}
In this paper we prove the fusion rules of Virasoro vertex operator algebras $L\left(c_{1, q}, 0\right)$, for $q \geq 1$. Roughly speaking, we consider $L\left(c_{1, q}, 0\right)$ as the limit of $L\left(c_{n, n q-1}, 0\right)$, for $n \rightarrow \infty$, and the fusion rules of $L\left(c_{1, q}, 0\right)$ follow as the limits of the fusion rules of $L\left(c_{n, n q-1}, 0\right)$.
\end{abstract}

\section{INTRODUCTION}

In classical representation theories (of compact groups or semi-simple Lie algebras), we can define the tensor product for two modules. The famous ClebschGordan problem is: given irreducible representations $U$ and $V$, describe the decomposition, with multiplicities, of the representation $U \otimes V$. For the representation theory of vertex operator algebras, the difference is that we cannot define the tensor product of two modules over a vertex operator algebra $A$. Nevertheless, we still have the analogue of the Clebsch-Gordan problem via the notion of intertwining operator. In particular, for three irreducible modules $U, V$ and $W$ over a vertex operator algebra $A$, we can define the fusion rule $\mathcal{N}_{U, V}^{W}$, the analogue of the ClebschGordan coefficient. For the representation theory of vertex operator algebras, it is also very important to determine the fusion rules $\mathcal{N}_{U, V}^{W}$.

The Virasoro vertex operator algebras constitute one of the most important classes of vertex operator algebras. In 15 it was proved that the Virasoro vertex operator algebras $L\left(c_{p, q}, 0\right)$ are rational, where $(p, q)=1, p, q>1$ and $c_{p, q}=$ $13-6\left(\frac{q}{p}+\frac{p}{q}\right)$. Furthermore, the fusion rules of $L\left(c_{p, q}, 0\right)$ were proved in [15] using the Frenkel-Zhu formula (cf. [7]). In the case of $L\left(c_{1, q}, 0\right)$, we cannot prove the fusion rules as in [15, for Frenkel-Zhu's formula cannot be applied to $L\left(c_{1, q}, 0\right)$ which is non-rational. The fusion rules of $L\left(c_{1,1}, 0\right)$ were first proved in [10] and further extended in 3 .

In this paper we prove the fusion rules of $L\left(c_{1, q}, 0\right)$, for $q \geq 1$. Our method is totally different from those of [3, 10]. Roughly speaking, we consider $L\left(c_{1, q}, 0\right)$ as the limit of $L\left(c_{n, n q-1}, 0\right)$, for $n \rightarrow \infty$, and the fusion rules of $L\left(c_{1, q}, 0\right)$ follow as the limits of the fusion rules of $L\left(c_{n, n q-1}, 0\right)$. Formally, the main result about fusion rules of $L\left(c_{1, q}, 0\right)$ can be written as

$$
L\left(c_{1, q}, h_{i_{1}, s_{1}}\right) \otimes L\left(c_{1, q}, h_{i_{2}, s_{2}}\right)=\bigoplus_{i \in A_{i_{1}, i_{2}} \in A_{s_{1}, s_{2}}} L\left(c_{1, q}, h_{i, s}\right),
$$

Received by the editors February 24, 2014 and, in revised form, May 2, 2014 and May 17, 2014. 2010 Mathematics Subject Classification. Primary 17B69.

Key words and phrases. Fusion rules, Virasoro vertex operator algebras, intertwining operator. This work was supported by the National Natural Science Foundation for young (no.11401098). 
where $A_{m, n}=\{m+n-1, m+n-3, \cdots,|m-n|+1\}$ for $m, n>0$. Many special cases of this result have already been applied in several papers (cf. [1,2,13]).

This paper is structured as follows: In Section 2 we give some preliminaries about the representation theory of Virasoro vertex operator algebras. In Section 3, using the easy part of Frenkel-Zhu's formula, we get an upper bound for the fusion rules of $L\left(c_{1, q}, 0\right)$. In Section 4 , we establish the fusion rules of $L\left(c_{1, q}, 0\right)$ by the limit method. In Section 5 , we further extend the fusion rules of $L\left(c_{1, q}, 0\right)$ to include some other cases. Throughout this paper, we assume that the reader is familiar with the axiom theory of vertex operator algebras and modules. For more information, see [6, 11.

\section{VERTEX OPERATOR ALGEBRAS AND MODULES ASSOCIATED TO VIRASORO ALGEBRA}

In this section, we give a short review of vertex operator algebras and modules associated to Virasoro algebra; details can be found in [4, 5, 8, 9, 11. First, recall that Virasoro algebra is the Lie algebra Vir with basis

$$
\left\{L_{n} \mid n \in \mathbb{Z}\right\} \cup\{C\},
$$

satisfying

and

$$
\left[L_{m}, L_{n}\right]=(m-n) L_{m+n}+\frac{m^{3}-m}{12} \delta_{m+n, 0} C
$$

$$
[V i r, C]=\{0\} \text {. }
$$

Define the following subalgebras of Vir:

$$
\begin{gathered}
V i r^{ \pm}=\bigoplus_{ \pm n>0} \mathbb{C} L_{n} ; V i r^{0}=L_{0} \oplus C ; \\
V i r^{\geq 0}=V i r^{+} \oplus V i r^{0} ; V i r^{\geq-1}=V i r^{\geq 0} \oplus \mathbb{C} L_{-1} .
\end{gathered}
$$

Let $c$ and $h$ be two complex numbers and let $\mathbb{C} v_{c, h}$ be the one dimensional $V i r \geq 0$ module with $C$ and $L_{0}$ acting respectively as the scalars $c$ and $h$, and with $\mathrm{Vir}^{+}$ acting trivially. Set

$$
M(c, h)=U(\text { Vir }) \otimes_{U(V i r \geq 0)} \mathbb{C} v_{c, h}
$$

and call it the Verma module with central charge $c$ and highest weight $h$. For any $\mathbb{I}=\left(1^{r_{1}} 2^{r_{2}} \cdots n^{r_{n}}\right) \in \mathcal{P}_{n}$, set

$$
e_{\mathbb{I}}=L_{-n}^{r_{n}} \cdots L_{-2}^{r_{2}} L_{-1}^{r_{1}} \in U\left(V_{i r}^{-}\right)_{-n} .
$$

Then, $\left\{e_{\mathbb{I}} \cdot v_{c, h} \mid \mathbb{I} \in \mathcal{P}_{n}\right\}$ forms a basis of the weight subspace $M(c, h)_{h+n}$. Let $M^{\prime}(c, h)$ be the largest proper submodule of $M(c, h)$. Then

$$
L(c, h)=M(c, h) / M^{\prime}(c, h)
$$

is an irreducible $V i r-$ module.

We recall the following proposition.

Proposition 2.1 (cf. [14]). Set $n=\alpha \beta, c=c(t)=13-6 t-6 t^{-1}$ and $h=h_{\alpha, \beta}(t)=$ $\frac{1}{4}\left(\alpha^{2}-1\right) t-\frac{1}{2}(\alpha \beta-1)+\frac{1}{4}\left(\beta^{2}-1\right) t^{-1}$ for $\alpha, \beta \in \mathbb{Z}_{>0}$. Then there exists

$$
S_{n}=\sum_{\mathbb{I} \in \mathcal{P}_{n}} f_{\mathbb{I}}(c, h) e_{\mathbb{I}} \in U\left(V i r^{-}\right)_{-n}
$$

such that $S_{n} v_{c, h} \in M(c, h)_{h+n}$ is a singular vector, where $f_{\mathbb{I}}(x, y) \in \mathbb{C}[x, y]$, and $f_{\mathbb{I}_{0}}(x, y)=1$ for $\mathbb{I}_{0}=\left(1^{n}\right)$. 
Let $p, q, r$ and $s$ be positive integers, satisfying $(p, q)=1, r<p$ and $s<q$. Let

$$
c=c_{p, q}=13-6\left(\frac{q}{p}+\frac{p}{q}\right)
$$

and

$$
h=h_{p, q ; r, s}=\frac{(s p-r q)^{2}-(p-q)^{2}}{4 p q} .
$$

Using Proposition 2.1, it can be shown that $M(c, h)$ has two singular vectors $u_{p, q}^{r, s}$ and $v_{p, q}^{r, s}$, of weights $h+r s$ and $h+(p-r)(q-s)$ respectively. Moreover, the maximal submodule of $M(c, h)$ is generated by $u_{p, q}^{r, s}$ and $v_{p, q}^{r, s}$.

Similarly, when $p=1$ and

$$
h=h_{i, s}=\frac{(i q-s)^{2}-(q-1)^{2}}{4 q}
$$

for some $i>0,0<s \leq q, M\left(c_{1, q}, h\right)$ has a singular vector of weight $h+i s$ which generates the maximal proper submodule of $M\left(c_{1, q}, h\right)$. Moreover, $M\left(c_{1, q}, h\right)=$ $L\left(c_{1, q}, h\right)$ when $h \neq h_{i, s}=\frac{(i q-s)^{2}-(q-1)^{2}}{4 q}$ for any $i>0,0<s \leq q$.

Now consider $\mathbb{C}$ as a $\operatorname{Vir} r^{\geq-1}$-module with $C$ acting as the scalar $c$, and with $\mathrm{Vir}^{+} \oplus L_{0} \oplus L_{-1}$ acting trivially. Set

$$
V_{c}=U(\text { Vir }) \otimes_{U(V i r \geq-1)} \mathbb{C} .
$$

It is well known that $V_{c}$ has a canonical structure of vertex operator algebra of central charge $c$ and with $\omega=L(-2) \mathbf{1}$ as conformal vector. In this way, $M(c, h)$ and $L(c, h)$ are modules for the vertex operator algebra $V_{c}$. Furthermore, $L(c, 0)$, as a quotient of $V_{c}$, is a simple vertex operator algebra.

Note that $L(c, 0)=V_{c}$ when $c \neq c_{p, q}$, where $p, q>1$ and $(p, q)=1$. If $c=c_{p, q}$ for some $p, q$ as above, then $L(c, 0) \neq V_{c}$, and $L(c, h)$ is an $L(c, 0)$-module if and only if $h=h_{p, q ; r, s}=\frac{(s p-r q)^{2}-(p-q)^{2}}{4 p q}$ for some positive integer $r, s$ satisfying $r<p$ and $s<q$ (cf. [15]). By the representation theory of Virasoro vertex operator algebras (cf. [11, 15]), we have

Theorem 2.2. $L\left(c_{1, q}, h\right)$ is an irreducible module for the vertex operator algebra $L\left(c_{1, q}, 0\right)$, and $L\left(c_{1, q}, h\right)$ for $h \in \mathbb{C}$ exhaust all the irreducible $L\left(c_{1, q}, 0\right)$-modules. For the vertex operator algebra $L\left(c_{p, q}, 0\right)(p, q>1$ and $(p, q)=1), L\left(c_{p, q}, h\right)$ for $h=h_{p, q ; r, s}=\frac{(s p-r q)^{2}-(p-q)^{2}}{4 p q} 0<r<p$ and $0<s<q$ exhaust all the irreducible $L\left(c_{p, q}, 0\right)$-modules.

Now we introduce the notions of intertwining operator and fusion rule for a triple of modules of vertex operator algebra (cf. [6]).

Definition 2.3. Let $W_{1}, W_{2}$ and $W_{3}$ be three modules over a vertex operator algebra $V$. A linear map $W_{1} \otimes W_{2} \rightarrow W_{3}\{x\}$, or equivalently,

$$
\begin{gathered}
W_{1} \rightarrow \operatorname{Hom}\left(W_{2}, W_{3}\right)\{x\} \\
w \mapsto \mathcal{Y}(w, x)=\sum_{n \in \mathbb{Q}} w_{n} x^{-n-1},
\end{gathered}
$$

where $w_{n} \in \operatorname{Hom}\left(W_{2}, W_{3}\right)$, is called an intertwining operator of type $\left(\begin{array}{c}W_{3} \\ W_{1} W_{2}\end{array}\right)$ if it satisfies:

1 (The truncation property) For any $w_{1} \in W_{1}, w_{2} \in W_{2}, w_{1}{ }_{n} w_{2}=0$ for $n$ sufficiently large; 
2 (The $L_{-1}$-derivative formula) For any $w \in W_{1}$,

$$
\mathcal{Y}\left(L_{-1} w, x\right)=\frac{d}{d x} \mathcal{Y}(w, x)
$$

3 (The Jacobi identity) For any $v \in V$ and $w_{1} \in W_{1}$,

$$
\begin{aligned}
& x_{0}^{-1} \delta\left(\frac{x_{1}-x_{2}}{x_{0}}\right) Y\left(v, x_{1}\right) \mathcal{Y}\left(w_{1}, x_{2}\right) \\
- & x_{0}^{-1} \delta\left(\frac{x_{2}-x_{1}}{-x_{0}}\right) \mathcal{Y}\left(w_{1}, x_{2}\right) Y\left(v, x_{1}\right) \\
= & x_{2}^{-1} \delta\left(\frac{x_{1}-x_{0}}{x_{2}}\right) \mathcal{Y}\left(Y\left(v, x_{0}\right) w_{1}, x_{2}\right) .
\end{aligned}
$$

Set $I\left(\begin{array}{c}W_{3} \\ W_{1} W_{2}\end{array}\right)$ to be the vector space of all intertwining operators of type $\left(\begin{array}{c}W_{3} \\ W_{1} W_{2}\end{array}\right)$. Its dimension $\mathcal{N}_{W_{1}, W_{2}}^{W_{3}}$ is called the fusion rule of type $\left(\begin{array}{c}W_{3} \\ W_{1} W_{2}\end{array}\right)$.

The main result of this paper is the following:

Theorem 2.4. Let $i_{n}>0,0<s_{n} \leq q(n=1,2,3)$, and $A_{m, n}=\{m+n-1, m+$ $n-3, \cdots,|m-n|+1\}$. Then

$$
\mathcal{N}_{L\left(c_{1, q}, h_{i_{1}, s_{1}}\right), L\left(c_{1, q}, h_{i_{2}, s_{2}}\right)}^{L\left(c_{1, q}, h_{i_{3}, s_{3}}\right)} \leq 1,
$$

and equality holds if and only if $h_{i_{3}, s_{3}}=h_{i, s}$ for some $i \in A_{i_{1}, i_{2}}$ and $s \in A_{s_{1}, s_{2}}$.

\section{Frenkel-Zhu's FORMUlA}

Recall that to a vertex operator algebra $V$, we can associate Zhu's algebra $A(V)$, and for each $V$-module $M$, the space of lowest weight vectors $M(0)$ has a natural structure of $A(V)$-module. More generally, for each $V$-module $M$, define $O(M) \subset$ $M$ to be the linear span of elements of type

$$
\operatorname{Res}_{z} Y(a, z) \frac{(1+z)^{\operatorname{deg} a}}{z^{2}} m
$$

where $a \in V$ and $m \in M$, and let $A(M)$ be the quotient space $M / O(M)$. Then $A(M)$ has a natural structure of an $A(V)$-bimodule. We recall the following useful result (cf. [7]).

Proposition 3.1. For each submodule $M_{1}$ of $M, A\left(M_{1}\right)$ is a submodule of the $A(V)$-bimodule $A(M)$, and the quotient $A(M) / A\left(M_{1}\right)$ is isomorphic to the bimodule $A\left(M / M_{1}\right)$.

For Virasoro vertex operator algebras and Verma modules, we have the following results (cf. [7,8]).

Proposition 3.2. Let $\mathcal{L}$ be the subalgebra of $\mathrm{Vir}^{-}$spanned by

$$
L_{-n-2}+2 L_{-n-1}+L_{-n}
$$

for $n \geq 1$. Then $O\left(V_{c}\right)=\mathcal{L} . V_{c}$ and $A\left(V_{c}\right) \cong H_{0}\left(\mathcal{L}, V_{c}\right)$. In the case of $M(c, h)$ (resp. the irreducible quotient $L(c, h)$ ), we also have

$$
\begin{gathered}
O(M(c, h))=\mathcal{L} \cdot M(c, h) \\
(\text { resp. } O(L(c, h))=\mathcal{L} \cdot L(c, h)),
\end{gathered}
$$

and

$$
\begin{gathered}
A(M(c, h)) \cong H_{0}(\mathcal{L}, M(c, h)) \\
\left(\text { resp. } A(L(c, h)) \cong H_{0}(\mathcal{L}, L(c, h))\right) .
\end{gathered}
$$


Proposition 3.3. We have an isomorphism of associative algebra:

$$
A\left(V_{c}\right) \cong \mathbb{C}[x] ; \quad[\omega]^{n} \mapsto x^{n}, \quad n \in \mathbb{Z}_{\geq 0} .
$$

For Verma module $M(c, h)$, the $A\left(V_{c}\right)$-bimodule $A(M(c, h))$ is isomorphic to $\mathbb{C}[x, y]$, where the highest weight vector $v_{c, h}$ represents $\mathbf{1} \in \mathbb{C}[x, y]$, and the left and the right actions of $A\left(V_{c}\right)$ are given by

$$
\begin{gathered}
x \cdot f(x, y)=x f(x, y), \\
f(x, y) \cdot x=y f(x, y),
\end{gathered}
$$

for any $f(x, y) \in \mathbb{C}[x, y]$.

Proposition 3.4. The left and right actions of $A\left(V_{c}\right)$ on $A(M(c, h))$ are given by

$$
\begin{gathered}
{[\omega][v]=\left[\left(L_{-2}+2 L_{-1}+L_{0}\right) v\right],} \\
{[v][\omega]=\left[\left(L_{-2}+L_{-1}\right) v\right],}
\end{gathered}
$$

for any $v \in M(c, h)$, where $\omega=L_{-2} \mathbf{1}$.

From now on, $W_{i}=\bigoplus_{n \in \mathbb{N}} W_{i}(n)(i=1,2,3)$ will always be irreducible $V$ modules, where $W_{i}(n)$ is the $L_{0}$-eigenspace of $W_{i}$ with eigenvalue $n+h_{i}$.

Proposition 3.5 (cf. [7]). Let $\mathcal{Y}(\cdot, x)$ be an intertwining operator of type $\left(\begin{array}{c}W_{3} \\ W_{1} W_{2}\end{array}\right)$. Then $\mathcal{Y}(\cdot, x)$ has the following form:

$$
\mathcal{Y}(w, x)=\sum_{n \in \mathbb{Z}} w(n) x^{-n-1} x^{-h_{1}-h_{2}+h_{3}},
$$

such that for any $w \in W_{1}(k)$,

$$
w(n) W_{2}(m) \subset W_{3}(m+k-n-1) .
$$

We also need the symmetry property of fusion rules, i.e., $\mathcal{N}_{W_{1}, W_{2}}^{W_{3}}=\mathcal{N}_{W_{2}, W_{1}}^{W_{3}}$ (cf. [6]).

Let $\mathcal{Y}(\cdot, x)$ be an intertwining operator of type $\left(\begin{array}{c}W_{3} \\ W_{1} W_{2}\end{array}\right)$. By Proposition 3.5 we can define a linear map oy from $W_{1} \otimes W_{2}(0)$ to $W_{3}(0)$ by sending $w_{1} \otimes w_{2}$ $\left(w_{1} \in W_{1}(n), w_{2} \in W_{2}(0)\right)$ to $w_{1}(n-1) w_{2}$. It can be proved that $w_{1}(n-1) w_{2}=0$ for $w_{1} \in O\left(W_{1}\right)$, and $o \mathcal{Y}$ induces an $A(V)$-homomorphism

$$
\pi(\mathcal{Y}): A\left(W_{1}\right) \otimes_{A(V)} W_{2}(0) \rightarrow W_{3}(0) .
$$

Thus we get a linear map:

$$
\pi: I\left(\begin{array}{c}
W_{3} \\
W_{1} W_{2}
\end{array}\right) \rightarrow \operatorname{Hom}_{A(V)}\left(A\left(W_{1}\right) \otimes_{A(V)} W_{2}(0), W_{3}(0)\right) .
$$

The Frenkel-Zhu formula (cf. 7) states that $\pi$ is an isomorphism if $W_{i}(i=1,2,3)$ are irreducible modules. It was pointed out in 12 that this formula only holds for rational vertex operator algebras, and for more general vertex operator algebras, we have the following proposition (cf. [12]).

Proposition 3.6. If $W_{3}$ is irreducible, then

$$
\pi: I\left(\begin{array}{c}
W_{3} \\
W_{1} W_{2}
\end{array}\right) \rightarrow \operatorname{Hom}_{A(V)}\left(A\left(W_{1}\right) \otimes_{A(V)} W_{2}(0), W_{3}(0)\right)
$$

is injective. 
Now we follow the arguments in [8, §9.3]. First, we consider the three $L\left(c_{1, q}, 0\right)$ modules $L\left(c_{1, q}, h_{i_{n}, s_{n}}\right)$, where $i_{n}>0,0<s_{n} \leq q(n=1,2,3)$. We want to compute the dimension of

$$
\operatorname{Hom}_{A\left(L\left(c_{1, q}, 0\right)\right)}\left(A\left(L\left(c_{1, q}, h_{i_{1}, s_{1}}\right)\right) \otimes_{A\left(L\left(c_{1, q}, 0\right)\right)} L\left(c_{1, q}, h_{i_{2}, s_{2}}\right)(0), L\left(c_{1, q}, h_{i_{3}, s_{3}}\right)(0)\right) .
$$

By Proposition 3.2, its dual space is isomorphic to the simultaneous eigenspace of the left and right actions of $[\omega]$ on $H_{0}\left(\mathcal{L}, L\left(c_{1, q}, h_{i_{1}, s_{1}}\right)\right)^{*}=H^{0}\left(\mathcal{L}, L\left(c_{1, q}, h_{i_{1}, s_{1}}\right)^{*}\right)$ with the eigenvalues $-h_{i_{3}, s_{3}}$ and $-h_{i_{2}, s_{2}}$ respectively; denote this eigenspace by $H^{0}\left(\mathcal{L}, L\left(c_{1, q}, h_{i_{1}, s_{1}}\right)^{*}\right)^{\left(-h_{i_{3}, s_{3}},-h_{i_{2}, s_{2}}\right)}$. Then the surjection

$$
M\left(c_{1, q}, h_{i_{1}, s_{1}}\right) \rightarrow L\left(c_{1, q}, h_{i_{1}, s_{1}}\right)
$$

induces an injection

$$
i: H^{0}\left(\mathcal{L}, L\left(c_{1, q}, h_{i_{1}, s_{1}}\right)^{*}\right)^{\left(-h_{i_{3}, s_{3}},-h_{i_{2}, s_{2}}\right)} \hookrightarrow H^{0}\left(\mathcal{L}, M\left(c_{1, q}, h_{i_{1}, s_{1}}\right)^{*}\right)^{\left(-h_{i_{3}, s_{3}},-h_{i_{2}, s_{2}}\right)} .
$$

The arguments in [8, §9.3] show that $H^{0}\left(\mathcal{L}, M\left(c_{1, q}, h_{i_{1}, s_{1}}\right)^{*}\right)^{\left(-h_{i_{3}, s_{3}},-h_{i_{2}, s_{2}}\right)}$ is one dimensional, and $i$ is an isomorphism if and only if

$$
P_{i_{1}, s_{1}}\left(-h_{i_{2}, s_{2}},-h_{i_{3}, s_{3}}+h_{i_{1}, s_{1}}, q\right)=0,
$$

where $P_{\alpha, \beta}(a, b ; \xi) \in \mathbb{C}\left[a, b, \xi, \xi^{-1}\right]$ satisfies

$$
P_{\alpha, \beta}(a, b ; \xi)^{2}=\prod_{k=0}^{\alpha-1} \prod_{l=0}^{\beta-1} Q_{k, l}^{\alpha, \beta}(a, b ; \xi),
$$

and

$$
\begin{aligned}
& Q_{k, l}^{\alpha, \beta}(a, b ; \xi) \\
& =\left[(b-a)-\left(k \xi^{\frac{1}{2}}-l \xi^{-\frac{1}{2}}\right)\left\{(\alpha-k) \xi^{\frac{1}{2}}-(\beta-l) \xi^{-\frac{1}{2}}\right\}\right] \\
& \times\left[(b-a)-\left\{(k+1) \xi^{\frac{1}{2}}-(l+1) \xi^{-\frac{1}{2}}\right\}\left\{(\alpha-k-1) \xi^{\frac{1}{2}}-(\beta-l-1) \xi^{-\frac{1}{2}}\right\}\right] \\
& +\left\{(\alpha-2 k-1) \xi^{\frac{1}{2}}-(\beta-l-1) \xi^{-\frac{1}{2}}\right\}^{2} a .
\end{aligned}
$$

Direct computation shows that equation (3.1) is equivalent to the equation

$$
\prod_{k=0}^{i_{1}-1} \prod_{l=0}^{s_{1}-1}\left(h_{i_{3}, s_{3}}-h_{i_{1}+i_{2}-2 k-1, s_{1}+s_{2}-2 l-1}\right)=0 .
$$

Now combining the symmetry property of fusion rules and Proposition 3.6 yields $\mathcal{N}_{L\left(c_{1, q}, h_{i_{1}, s_{1}}\right), L\left(c_{1, q}, h_{i_{2}, s_{2}}\right)}^{L\left(c_{1,}, h_{i_{3}, s_{3}}\right)} \leq 1$, and $\mathcal{N}_{L\left(c_{1, q}, h_{i_{1}, s_{1}}\right), L\left(c_{1, q}, h_{i_{2}, s_{2}}\right)}^{L\left(c_{1, q}, h_{i_{3}, s_{3}}\right)}=1$ only if the following two equations hold:

$$
\begin{aligned}
& \prod_{k=0}^{i_{1}-1} \prod_{l=0}^{s_{1}-1}\left(h_{i_{3}, s_{3}}-h_{i_{1}+i_{2}-2 k-1, s_{1}+s_{2}-2 l-1}\right)=0 \\
& \prod_{k=0}^{i_{2}-1} \prod_{l=0}^{s_{2}-1}\left(h_{i_{3}, s_{3}}-h_{i_{1}+i_{2}-2 k-1, s_{1}+s_{2}-2 l-1}\right)=0 .
\end{aligned}
$$

For $m, n>0$, set $A_{m, n}=\{m+n-1, m+n-3, \cdots,|m-n|+1\}$. Direct computation shows that these two equations are equivalent to the existences of $i \in A_{i_{1}, i_{2}}, s \in$ $A_{s_{1}, s_{2}}$ such that $h_{i_{3}, s_{3}}=h_{i, s}$. 
To sum up, we have proved that $\mathcal{N}_{L\left(c_{1, q}, h_{i_{1}, s_{1}}\right), L\left(c_{1, q}, h_{i_{2}, s_{2}}\right)}^{L\left(c_{1, q}, h_{i_{3}, s_{3}}\right)} \leq 1$ if there exists $i \in A_{i_{1}, i_{2}}, s \in A_{s_{1}, s_{2}}$ such that $h_{i_{3}, s_{3}}=h_{i, s}$, and $\mathcal{N}_{L\left(c_{1, q}, h_{i_{1}, s_{1}}\right), L\left(c_{1, q}, h_{i_{2}, s_{2}}\right)}^{L\left(c_{1, q}, h_{\left.i_{3}, s_{3}\right)}\right.}=0$ otherwise.

\section{Construction of intertwining operators}

We fix three $L\left(c_{1, q}, 0\right)$-modules $L\left(c_{1, q}, h_{i_{n}, s_{n}}\right)(n=1,2,3)$, where $i_{n}>0$, $0<s_{n} \leq q$, and assume that there exist $i \in A_{i_{1}, i_{2}}, s \in A_{s_{1}, s_{2}}$, satisfying $h_{i_{3}, s_{3}}=$ $h_{i, s}$. The purpose of this section is to construct a non-zero intertwining operator of type $\left(\begin{array}{c}L\left(c_{1, q}, h_{i_{3}, s_{3}}\right) \\ L\left(c_{1, q}, h_{i_{1}, s_{1}}\right) L\left(c_{1, q}, h_{i_{2}, s_{2}}\right)\end{array}\right)$. Set $c_{k}=c_{k, k q-1}$, and $h_{n}^{k}=\frac{\left(i_{n}(k q-1)-s_{n} k\right)^{2}-(k q-1-k)^{2}}{4 k(k q-1)}$.

Lemma 4.1. $\lim _{k \rightarrow \infty} c_{k}=c_{1, q}$, and $\lim _{k \rightarrow \infty} h_{n}^{k}=h_{i_{n}, s_{2}}$, for $n=1,2,3$.

By the fusion rules of $L\left(c_{k}, 0\right)$ when $k$ is large enough, there exists a non-trivial intertwining operator $\mathcal{Y}_{k}(\cdot, x)$ of type $\left(\begin{array}{c}L\left(c_{k}, h_{3}^{k}\right) \\ L\left(c_{k}, h_{1}^{k}\right) L\left(c_{k}, h_{2}^{k}\right)\end{array}\right)$. Our method is to get the desired intertwining operator from the limit of $\mathcal{Y}_{k}(\cdot, x)$ as $k$ approaches infinity. Hence, from now on, we always assume that $k$ is large enough when needed. We say that a sequence of monomials $\left\{a_{k} x^{n_{k}}\right\}$ converges to the limit $a x^{n}$

$$
\lim _{k \rightarrow \infty} a_{k} x^{n_{k}}=a x^{n}
$$

if $\left\{a_{k}\right\}$ and $\left\{n_{k}\right\}$ converge to the limits $a$ and $n$ respectively. The following proposition is crucial for our construction.

Proposition 4.2. As a left $A\left(L\left(c_{k}, 0\right)\right)$-module, $A\left(L\left(c_{k}, h_{n}^{k}\right)\right)$ is generated by

$$
\left[v_{c_{k}, h_{n}^{k}}\right],\left[L_{-1} v_{c_{k}, h_{n}^{k}}\right], \cdots,\left[L_{-1}^{i_{n} s_{n}-1} v_{c_{k}, h_{n}^{k}}\right] .
$$

Proof. Combining Propositions 3.2, 3.3 and 3.4 implies

$$
\left[L_{-n} v\right]=(-1)^{n}(n y-x+w t(v))[v]
$$

in $A\left(M\left(c_{k}, h_{n}^{k}\right)\right)$ for each homogenous $v \in L\left(c_{k}, h_{n}^{k}\right)$ (recall the identification $A\left(M\left(c_{k}, h_{n}^{k}\right)\right)=\mathbb{C}[x, y]$ in Proposition 3.3). By this formula and Proposition 2.1. we have

$$
\left[L_{-1}^{m} v_{c_{k}, h_{n}^{k}}\right]=(x-y)^{m}+\text { lower powers of } x \text { and } y
$$

and

$$
\left[S_{i_{n} s_{n}} v_{c_{k}, h_{n}^{k}}\right]=\left[v_{i_{n}, s_{n}}\right]=(x-y)^{i_{n} s_{n}}+\text { lower powers of } x \text { and } y
$$

in $A\left(M\left(c_{k}, h_{n}^{k}\right)\right) \cong \mathbb{C}[x, y]$. Now by Proposition [3.1, $\left[S_{i_{n} s_{n}} v_{c_{k}, h_{n}^{k}}\right]$ lies in the kernel of the surjective morphism $A\left(M\left(c_{k}, h_{n}^{k}\right)\right) \rightarrow A\left(L\left(c_{k}, h_{n}^{k}\right)\right)$, hence $A\left(L\left(c_{k}, h_{n}^{k}\right)\right)$ can be generated, as a left $A\left(L\left(c_{k}, 0\right)\right)$-module by

$$
\left[v_{c_{k}, h_{n}^{k}}\right],\left[L_{-1} v_{c_{k}, h_{n}^{k}}\right], \cdots,\left[L_{-1}^{i_{n} s_{n}-1} v_{c_{k}, h_{n}^{k}}\right] .
$$

Now we are well prepared for the construction. It suffices to construct a bilinear pair $\lceil\cdot, \cdot\rfloor$ (taking values in $\mathbb{C}\{x\})$ between $\left(L\left(c_{1, q}, h_{i_{3}, s_{3}}\right)\right)^{*}$ and $L\left(c_{1, q}, h_{i_{1}, s_{1}}\right) \otimes$ $L\left(c_{1, q}, h_{i_{2}, s_{2}}\right)$ that satisfies the corresponding properties. The construction is divided into several steps. Throughout the construction, we adopt the following convention:

We always identify $M\left(c_{1, q}, h_{i_{n}, s_{n}}\right)(n=1,2,3)$ with $M\left(c_{k}, h_{n}^{k}\right)$, for $k \geq 1$, by isomorphisms which send $v_{c_{1, q}, h_{i_{n}, s_{n}}}$ to $v_{c_{k}, h_{n}^{k}}$ and commute with the action of $\mathrm{Vir}^{-}$. 
For a $V i r-$ module $M$ of lowest weight $h$, we always use $M(n)$ to denote the subspace of vectors of weight $h+n$.

Step 1. Let $v^{\prime} \in L\left(c_{1, q}, h_{i_{3}, s_{3}}\right)^{*}(0)=M\left(c_{1, q}, h_{i_{3}, s_{3}}\right)^{*}(0)=M\left(c_{k}, h_{3}^{k}\right)^{*}(0)$ be defined by $v^{\prime}\left(v_{c_{1, q}, h_{i_{3}, s_{3}}}\right)=1$. For each homogenous $v_{1} \in M\left(c_{1, q}, h_{i_{1}, s_{1}}\right)=M\left(c_{k}, h_{1}^{k}\right)$ and $v_{2}=v_{c_{1, q}, h_{i_{2}, s_{2}}}=v_{c_{k}, h_{2}^{k}},\left\lceil v^{\prime}, v_{1} \otimes v_{2}\right\rfloor$ is defined as follows:

Set $a_{1}=v_{c_{1, q}, h_{i_{1}, s_{1}}}, a_{2}=L_{-1} v_{c_{1, q}, h_{i_{1}, s_{1}}}, \cdots, a_{i_{1} s_{1}}=L_{-1}^{i_{1} s_{1}-1} v_{c_{1, q}, h_{i_{1}, s_{1}}}$ in $M\left(c_{1, q}, h_{i_{1}, s_{1}}\right)=M_{c_{k}, h_{1}^{k}}$. Then for each $k$, there is some $i$ such that $\left\langle v^{\prime}, \mathcal{Y}_{k}\left(a_{i}, x\right) v_{2}\right\rangle$ $\neq 0$. Otherwise, by Propositions 3.6 and 4.2 $\mathcal{Y}_{k}$ will be zero. Since $\left\langle v^{\prime}, \mathcal{Y}_{k}\left(a_{i+1}, x\right) v_{2}\right\rangle$ is the derivation of $\left\langle v^{\prime}, \mathcal{Y}_{k}\left(a_{i}, x\right) v_{2}\right\rangle$, we have $\left\langle v^{\prime}, \mathcal{Y}_{k}\left(a_{1}, x\right) v_{2}\right\rangle \neq 0$ for each $k$. By Proposition 3.5 and Lemma 4.1, when replacing each $\mathcal{Y}_{k}$ by a suitable non-zero multiple,

$$
\left\langle v^{\prime}, \mathcal{Y}_{1}\left(a_{1}, x\right) v_{2}\right\rangle,\left\langle v^{\prime}, \mathcal{Y}_{2}\left(a_{1}, x\right) v_{2}\right\rangle, \cdots,\left\langle v^{\prime}, \mathcal{Y}_{k}\left(a_{1}, x\right) v_{2}\right\rangle, \cdots
$$

converges to a non-zero monomial. Now assume that the sequence

$$
\left\langle v^{\prime}, \mathcal{Y}_{1}(v, x) v_{2}\right\rangle,\left\langle v^{\prime}, \mathcal{Y}_{2}(v, x) v_{2}\right\rangle, \cdots,\left\langle v^{\prime}, \mathcal{Y}_{k}(v, x) v_{2}\right\rangle, \cdots
$$

converges for a homogeneous $v \in M\left(c_{1, q}, h_{i_{1}, s_{1}}\right)$. Then the same result holds for $L_{0} v$ and $L_{-1} v$. As the left action of $A\left(V_{c}\right)$ on $A(M(c, h))$ is given by

$$
[\omega][v]=\left[\left(L_{-2}+2 L_{-1}+L_{0}\right) v\right],
$$

by the construction of the linear map $\pi$ in Section 3, we see that

$$
\left\langle v^{\prime}, \mathcal{Y}_{1}\left(L_{-2} v, x\right) v_{2}\right\rangle,\left\langle v^{\prime}, \mathcal{Y}_{2}\left(L_{-2} v, x\right) v_{2}\right\rangle, \cdots,\left\langle v^{\prime}, \mathcal{Y}_{k}\left(L_{-2} v, x\right) v_{2}\right\rangle, \cdots
$$

also converges. By induction and the equality $\left[L_{-n}, L_{-1}\right]=(1-n) L_{-n-1}$, the same is true for $L_{-n} v(n>2)$. Since $M\left(c_{1, q}, h_{i_{1}, s_{1}}\right)$ is generated by $a_{1}=v_{c_{1, q}, h_{i_{1}}, s_{1}}$, we conclude by induction that for each homogeneous $v_{1} \in M\left(c_{1, q}, h_{i_{1}, s_{1}}\right)$, the sequence

$$
\left\langle v^{\prime}, \mathcal{Y}_{1}\left(v_{1}, x\right) v_{2}\right\rangle,\left\langle v^{\prime}, \mathcal{Y}_{2}\left(v_{1}, x\right) v_{2}\right\rangle, \cdots,\left\langle v^{\prime}, \mathcal{Y}_{k}\left(v_{1}, x\right) v_{2}\right\rangle, \cdots
$$

converges. Let $\left\lceil v^{\prime}, v_{1} \otimes v_{2}\right\rfloor$ be the limit and Step 1 is complete.

Step 2. For any $v_{1} \in M\left(c_{1, q}, h_{i_{1}, s_{1}}\right)$ and $v_{2} \in M\left(c_{1, q}, h_{i_{2}, s_{2}}\right)$ we want to define $\left\lceil v^{\prime}, v_{1} \otimes v_{2}\right\rfloor$ as the limit of the sequence

$$
\left\langle v^{\prime}, \mathcal{Y}_{1}\left(v_{1}, x\right) v_{2}\right\rangle,\left\langle v^{\prime}, \mathcal{Y}_{2}\left(v_{1}, x\right) v_{2}\right\rangle, \cdots,\left\langle v^{\prime}, \mathcal{Y}_{k}\left(v_{1}, x\right) v_{2}\right\rangle, \cdots .
$$

Thus we need to show that the limit of the sequence (4.1) exists for each $v_{1} \in$ $M\left(c_{1, q}, h_{i_{1}, s_{1}}\right)$ and $v_{2} \in M\left(c_{1, q}, h_{i_{2}, s_{2}}\right)$. Step 1 shows that when $v_{2}=v_{c_{1, q}, h_{i_{2}, s_{2}}}$, the limit exists. Thus by induction, it suffices to prove that if the limit of the sequence (4.1) exists for any $v_{1} \in M\left(c_{1, q}, h_{i_{1}, s_{1}}\right)$ and a fixed $v_{2} \in M\left(c_{1, q}, h_{i_{2}, s_{2}}\right)$, then the same is true with $v_{2}$ replaced by $L_{n} v_{2}(n \leq-1)$. But this follows directly from the following identity:

$$
\begin{array}{r}
\left\langle v^{\prime}, \mathcal{Y}_{k}\left(v_{1}, x\right) L_{n} v_{2}\right\rangle=\left\langle v^{\prime}, L_{n} \mathcal{Y}_{k}\left(v_{1}, x\right) v_{2}\right\rangle \\
-\sum_{i=0}^{\infty}\left(\begin{array}{c}
n+1 \\
i
\end{array}\right) x^{n+1-i}\left\langle v^{\prime}, \mathcal{Y}_{k}\left(L_{i-1} v_{1}, x\right) v_{2}\right\rangle \\
=-\sum_{i=0}^{\infty}\left(\begin{array}{c}
n+1 \\
i
\end{array}\right) x^{n+1-i}\left\langle v^{\prime}, \mathcal{Y}_{k}\left(L_{i-1} v_{1}, x\right) v_{2}\right\rangle
\end{array}
$$


Hence we conclude that the limit of the sequence (4.1) exists for any homogeneous $v_{2} \in M\left(c_{1, q}, h_{i_{2}, s_{2}}\right)$. Set

$$
\left\lceil v^{\prime}, v_{1} \otimes v_{2}\right\rfloor=\lim _{k \rightarrow \infty}\left\langle v^{\prime}, \mathcal{Y}_{k}\left(v_{1}, x\right) v_{2}\right\rangle,
$$

and Step 2 is complete.

Step 3. Now we want to define $\left\lceil v_{3}^{\prime}, v_{1} \otimes v_{2}\right\rfloor$ for any homogeneous $v_{1} \in M\left(c_{1, q}, h_{i_{1}, s_{1}}\right)$, $v_{2} \in M\left(c_{1, q}, h_{i_{2}, s_{2}}\right)$ and $v_{3}^{\prime} \in L\left(c_{1, q}, h_{i_{3}, s_{3}}\right)^{*} \subset M\left(c_{1, q}, h_{i_{3}, s_{3}}\right)^{*}$. By Lemma 4.1, we have

Lemma 4.3. Given a positive integer $m$ and a homogeneous $v \in M\left(c_{1, q}, h_{i_{n}, s_{n}}\right)=$ $M\left(c_{k}, h_{n}^{k}\right), L_{m} v$ as a vector in $M\left(c_{k}, h_{n}^{k}\right)$, converges to $L_{m} v$ as a vector in $M\left(c_{1, q}, h_{i_{n}, s_{n}}\right)$, when $k$ approaches infinity.

Lemma 4.4. For any $\mathbb{I} \in \mathcal{P}_{n},\left\langle e_{\mathbb{I}} v^{\prime}, \mathcal{Y}_{k}\left(v_{1}, x\right) v_{2}\right\rangle$ converges to a finite limit as $k$ approaches infinity, where $e_{\mathbb{I}} v^{\prime} \in M\left(c_{k}, h_{3}^{k}\right)$.

Proof. By induction on the length of $\mathbb{I}$, this lemma follows directly from Lemma 4.3 and the formula

$$
\left\langle L_{n} w^{\prime}, w\right\rangle=\left\langle w^{\prime}, L_{-n} w\right\rangle
$$

for any $w^{\prime} \in L\left(c_{k}, h_{3}^{k}\right)^{*}$ and $w \in L\left(c_{k}, h_{3}^{k}\right)$.

Since $L\left(c_{1, q}, h_{i_{3}, s_{3}}\right)$ is irreducible, $L\left(c_{1, q}, h_{i_{3}, s_{3}}\right)^{*}$ is generated by $L\left(c_{1, q}, h_{i_{3}, s_{3}}\right)^{*}(0)$ $=\mathbb{C} v^{\prime}$ as a module over $V i r^{-}$. Hence we can choose a subset $\left\{\mathbb{I}_{1}, \cdots, \mathbb{I}_{s}\right\}$ of $\mathcal{P}_{n}$ such that $e_{\mathbb{I}_{1}} v^{\prime}, \cdots, e_{\mathbb{I}_{s}} v^{\prime}$ form a basis of $L\left(c_{1, q}, h_{i_{3}, s_{3}}\right)^{*}(n)$. By our convention, both $L\left(c_{1, q}, h_{i_{3}, s_{3}}\right)^{*}(n)$ and $L\left(c_{k}, h_{3}^{k}\right)^{*}(n)$ are subspaces of $M\left(c_{1, q}, h_{i_{3}, s_{3}}\right)^{*}(n)=$ $M\left(c_{k}, h_{3}^{k}\right)^{*}(n)$. Moreover, by Proposition 2.1 and Lemma 4.1.

$$
\lim _{k \rightarrow \infty} L\left(c_{k}, h_{3}^{k}\right)^{*}(n)=L\left(c_{1, q}, h_{i_{3}, s_{3}}\right)^{*}(n),
$$

where dimensions of $L\left(c_{k}, h_{3}^{k}\right)^{*}(n)$ and $L\left(c_{1, q}, h_{i_{3}, s_{3}}\right)^{*}(n)$ are equal for large enough $k$ and the limit is taken with respect to the topology of complex Grassmanian. Hence it is easy to see that $e_{\mathbb{I}_{1}} v^{\prime}, \cdots, e_{\mathbb{I}_{s}} v^{\prime}$, as elements of $M\left(c_{k}, h_{3}^{k}\right)^{*}$, form a basis of $L\left(c_{k}, h_{3}^{k}\right)^{*}(n)$, and converge to $e_{\mathbb{I}_{1}} v^{\prime}, \cdots, e_{\mathbb{I}_{s}} v^{\prime}$ respectively in $M\left(c_{1, q}, h_{i_{3}, s_{3}}\right)^{*}$ as $k$ approaches infinity.

Now for any homogeneous $v_{3}^{\prime} \in L\left(c_{1, q}, h_{i_{3}, s_{3}}\right)^{*}(n)$ we can choose a

$$
v_{3, k}^{\prime} \in L\left(c_{k}, h_{3}^{k}\right)^{*}(n)
$$

for each $k$, such that the sequence $\left\{v_{3, k}^{\prime}\right\}$ converges to $v_{3}^{\prime}$. If we write

$$
v_{3}^{\prime}=a_{1} e_{\mathbb{I}_{1}} v^{\prime}+\cdots+a_{s} e_{\mathbb{I}_{s}} v^{\prime}
$$

and

$$
v_{3, k}^{\prime}=a_{1, k} e_{\mathbb{I}_{1}} v^{\prime}+\cdots+a_{s, k} e_{\mathbb{I}_{s}} v^{\prime},
$$

then for each $i$, the sequence

$$
\cdots a_{i, k}, a_{i, k+1}, a_{i, k+2} \cdots
$$

converges to $a_{i}$. By Lemma 4.4 we can set

$$
\left\lceil v_{3}^{\prime}, v_{1} \otimes v_{2}\right\rfloor=\lim _{k \rightarrow \infty}\left\langle v_{3, k}^{\prime}, \mathcal{Y}_{k}\left(v_{1}, x\right) v_{2}\right\rangle .
$$

One checks that it is independent of the choice of the sequence $\left\{v_{3, k}^{\prime}\right\}$. 
Step 4. Now we check that the pairing $\lceil\cdot, \cdot\rfloor$ induces an intertwining operator of type $\left(\begin{array}{c}L\left(c_{1, q}, h_{i_{3}, s_{3}}\right) \\ M\left(c_{1, q}, h_{i_{1}, s_{1}}\right) M\left(c_{1, q}, h_{i_{2}, s_{2}}\right)\end{array}\right)$.

It suffices to verify the $L_{-1}$-derivative formula

$$
\frac{d}{d x}\left\lceil v_{3}^{\prime}, v_{1} \otimes v_{2}\right\rfloor=\left\lceil v_{3}^{\prime}, L_{-1} v_{1} \otimes v_{2}\right\rfloor
$$

and the Jacobi identity

$$
\begin{aligned}
x_{0}^{-1} \delta\left(\frac{x_{1}-x}{x_{0}}\right)\left\lceil Y\left(e^{x_{1} L_{1}}\left(-x_{1}^{-2}\right)^{L_{0}} v, x_{1}^{-1}\right) v_{3}^{\prime}, v_{1} \otimes v_{2}\right\rfloor \\
-x_{0}^{-1} \delta\left(\frac{x-x_{1}}{-x_{0}}\right)\left\lceil v_{3}^{\prime}, v_{1} \otimes Y\left(v, x_{1}\right) v_{2}\right\rfloor \\
=x^{-1} \delta\left(\frac{x_{1}-x_{0}}{x}\right)\left\lceil v_{3}^{\prime}, Y\left(v, x_{1}\right) v_{1} \otimes v_{2}\right\rfloor,
\end{aligned}
$$

where $v \in V_{c_{1, q}}$ (this form of Jacobi identity follows from the graded dual module structure of $L\left(c_{1, q}, h_{i_{3}, s_{3}}\right)^{*}$ defined in $\S 5.2$ of [6]), and the truncation property follows as a consequence.

The $L_{-1}$-derivative formula follows directly from our definition of $\lceil\cdot, \cdot\rfloor$ and the fact that the derivation $\frac{d}{d x}$ commutes with the limiting operation. In order to prove the Jacobi identity, we identify $V_{c_{1, q}}$ with $V_{c_{k}}$ by linear isomorphism $V_{c_{1, q}} \rightarrow V_{c_{k}}$ which sends 1 to 1 and commutes with the action of $V i r^{-}$. Then by Lemma 4.1 the coefficients of $Y\left(v, x_{0}\right) v_{1}$ (resp. $Y\left(v, x_{1}\right) v_{2}$ ), as elements of $M\left(c_{k}, h_{1}^{k}\right)$ (resp. $M\left(c_{k}, h_{2}^{k}\right)$ ), converge to the corresponding coefficients of $Y\left(v, x_{0}\right) v_{1}$ (resp. $\left.Y\left(v, x_{1}\right) v_{2}\right)$, as elements of $M\left(c_{1, q}, h_{i_{1}, s_{1}}\right)$ (resp. $\left.M\left(c_{1, q}, h_{i_{2}, s_{2}}\right)\right)$. If we choose, as in Step 3, a $v_{3, k}^{\prime} \in L\left(c_{k}, h_{3}^{k}\right)^{*}$ for each $k$, such that the sequence $\left\{v_{3, k}^{\prime}\right\}$ converges to $v_{3}^{\prime}$, then the coefficients of $Y\left(e^{x_{1} L_{1}}\left(-x_{1}^{-2}\right)^{L_{0}} v, x_{1}^{-1}\right) v_{3, k}^{\prime}$ converge to the corresponding coefficients of $Y\left(e^{x_{1} L_{1}}\left(-x_{1}^{-2}\right)^{L_{0}} v, x_{1}^{-1}\right) v_{3}^{\prime}$. Now the Jacobi identity of $\lceil\cdot, \cdot\rfloor$ follows from the Jacobi identities of $\mathcal{Y}_{k}$ and Step 4 is complete.

Step 5. Show that $\left\lceil v_{3}^{\prime}, v_{1} \otimes v_{2}\right\rfloor=0$ if $v_{1}$ lies in the maximal proper submodule $M^{\prime}\left(c_{1, q}, h_{i_{1}, s_{1}}\right)$ of $M\left(c_{1, q}, h_{i_{1}, s_{1}}\right)$. Let $M^{\prime}\left(c_{k}, h_{1}^{k}\right)$ be the maximal submodule of $M\left(c_{k}, h_{1}^{k}\right)$; then it is easy to see that $M^{\prime}\left(c_{k}, h_{1}^{k}\right)(n)$ converges to $M^{\prime}\left(c_{1, q}, h_{i_{1}, s_{1}}\right)(n)$ for each $n$ as $k$ approaches infinity. Thus there exists a $v_{1, k} \in M^{\prime}\left(c_{k}, h_{1}^{k}\right)$ for each $k$, such that the sequence $\left\{v_{1, k}\right\}$ converges to $v_{1}$. Using the arguments of Step 3, we see that

$$
\left\lceil v_{3}^{\prime}, v_{1} \otimes v_{2}\right\rfloor=\lim _{k \rightarrow \infty}\left\langle v_{3, k}^{\prime}, \mathcal{Y}_{k}\left(v_{1, k}, x\right) v_{2}\right\rangle
$$

where $\left\{v_{3, k}^{\prime}\right\}$ is the sequence converging to $v_{3}^{\prime}$ in Step 3. Since $\mathcal{Y}_{k}(\cdot, x)$ is an intertwining operator of type $\left(\begin{array}{c}L\left(c_{k}, h_{3}^{k}\right) \\ L\left(c_{k}, h_{1}^{k}\right) L\left(c_{k}, h_{2}^{k}\right)\end{array}\right)$, this forces $\left\lceil v_{3}^{\prime}, v_{1} \otimes v_{2}\right\rfloor=0$.

Step 6. Show that $\left\lceil v_{3}^{\prime}, v_{1} \otimes v_{2}\right\rfloor=0$ if $v_{2}$ lies in the maximal proper submodule $M^{\prime}\left(c_{1, q}, h_{i_{2}, s_{2}}\right)$ of $M\left(c_{1, q}, h_{i_{2}, s_{2}}\right)$. It suffices to repeat the arguments of Step 5 and we omit the details.

From the above construction we see that the pairing $\lceil\cdot, \cdot\rfloor$ induces a non-zero intertwining operator $\mathcal{Y}(\cdot, x)$ of type $\left(\begin{array}{c}L\left(c_{1, q}, h_{i_{3}, s_{3}}\right) \\ L\left(c_{1, q}, h_{i_{1}, s_{1}}\right) L\left(c_{1, q}, h_{i_{2}, s_{2}}\right)\end{array}\right)$ such that $\left\lceil v_{3}^{\prime}, v_{1} \otimes\right.$ $\left.v_{2}\right\rfloor=\left\langle v_{3}^{\prime}, \mathcal{Y}\left(v_{1}, x\right) v_{2}\right\rangle$. This finishes our construction. Hence the proof of Theorem 2.4 is complete. 
Remark 4.5. The limit method is quite necessary, for we cannot construct these intertwining operators by lattice vertex operator algebras as in [3,10. We hope to formalize this method and find more applications in future work.

\section{FurTher EXTENSION}

From Section 2, we see that the Verma module $M\left(c_{1, q}, h\right)$ is irreducible if and only if $h \neq h_{i, s}=\frac{(i q-s)^{2}-(q-1)^{2}}{4 q}$ for any $i>0,0<s \leq q$. In this section, we consider the fusion rules of the type

$$
\left(\begin{array}{c}
M\left(c_{1, q}, h^{\prime}\right) \\
L\left(c_{1, q}, h_{i_{1}, s_{1}}\right) M\left(c_{1, q}, h\right)
\end{array}\right) \quad \text { and } \quad\left(\begin{array}{c}
M\left(c_{1, q}, h\right) \\
L\left(c_{1, q}, h_{i_{1}, s_{1}}\right) L\left(c_{1, q}, h_{i_{2}, s_{2}}\right)
\end{array}\right)
$$

where $M\left(c_{1, q}, h\right)$ and $M\left(c_{1, q}, h^{\prime}\right)$ are irreducible Verma modules and $i_{1}, s_{1}, i_{2}, s_{2}$ are as before. By Theorem 2.11 in [12, Frenkel-Zhu's formula holds in the first case. Hence the argument of Section 3 directly implies

Theorem 5.1.

$$
\mathcal{N}_{L\left(c_{1, q}, h_{i, s}\right), M\left(c_{1, q}, h\right)}^{M\left(c_{1, q}, h^{\prime}\right)} \leq 1
$$

where $i>0,0<s \leq q$, and $M\left(c_{1, q}, h\right)$ and $M\left(c_{1, q}, h^{\prime}\right)$ are irreducible Verma modules. Set $h=\frac{s^{\prime 2}-(q-1)^{2}}{4 q}$ for some complex number $s^{\prime}$. Then the equality holds if and only if $h^{\prime}=\frac{\left(j q-s^{\prime}-t\right)^{2}-(q-1)^{2}}{4 q}$ for some $j \in\{-i+1,-i+3, \cdots, i-1\}$ and $t \in\{-s+1,-s+3, \cdots, s-1\}$.

Remark 5.2. As in [10], Theorem 5.1 can also be proved by considering $M\left(c_{1, q}, h\right)$ and $M\left(c_{1, q}, h^{\prime}\right)$ as modules over lattice vertex operator algebra.

Similarly, we have

\section{Theorem 5.3.}

$$
\mathcal{N}_{L\left(c_{1, q}, h_{i_{1}, s_{1}}\right), L\left(c_{1, q}, h_{i_{2}, s_{2}}\right)}^{M\left(c_{1, q}, h\right)}=\mathcal{N}_{L\left(c_{1, q}, h_{i_{1}, s_{1}}\right), M\left(c_{1, q}, h\right)}^{L\left(c_{1,}, h_{i_{2}, s_{2}}\right)}=0
$$

where $i_{1}, i_{2}>0,0<s_{1}, s_{2} \leq q$ and $M\left(c_{1, q}, h\right)$ is an irreducible Verma module.

\section{ACKnowledgments}

The author would like to express his deep gratitude to A. Milas for valuable comments and suggestions about this paper.

\section{REFERENCES}

[1] Dražen Adamović and Antun Milas, Logarithmic intertwining operators and $\mathcal{W}(2,2 p-1)$ algebras, J. Math. Phys. 48 (2007), no. 7, 073503, 20, DOI 10.1063/1.2747725. MR.2337684 (2009b:81079)

[2] Dražen Adamović and Antun Milas, On the triplet vertex algebra $\mathcal{W}(p)$, Adv. Math. 217 (2008), no. 6, 2664-2699, DOI 10.1016/j.aim.2007.11.012. MR2397463(2009e:17050)

[3] Chongying Dong and Cuipo Jiang, A characterization of vertex operator algebra $L\left(\frac{1}{2}, 0\right) \otimes$ $L\left(\frac{1}{2}, 0\right)$, Comm. Math. Phys. 296 (2010), no. 1, 69-88, DOI 10.1007/s00220-009-0964-4. MR2606628 (2011c:17049)

[4] B. L. Feigin and D. B. Fuks, Representation of the Virasoro algebra, in: Representations of Infinite Dimensional Lie Groups and Lie Algebras, Gordon and Breach, 1989.

[5] B. L. Fergin and D. B. Fuchs, Verma modules over the Virasoro algebra, Topology (Leningrad, 1982), Lecture Notes in Math., vol. 1060, Springer, Berlin, 1984, pp. 230-245, DOI 10.1007/BFb0099939. MR.770243 (86g:17004) 
[6] Igor B. Frenkel, Yi-Zhi Huang, and James Lepowsky, On axiomatic approaches to vertex operator algebras and modules, Mem. Amer. Math. Soc. 104 (1993), no. 494, viii+64, DOI 10.1090/memo/0494. MR.1142494 (94a:17007)

[7] Igor B. Frenkel and Yongchang Zhu, Vertex operator algebras associated to representations of affine and Virasoro algebras, Duke Math. J. 66 (1992), no. 1, 123-168, DOI 10.1215/S00127094-92-06604-X. MR1159433 (93g:17045)

[8] Kenji Iohara and Yoshiyuki Koga, Representation theory of the Virasoro algebra, Springer Monographs in Mathematics, Springer-Verlag London, Ltd., London, 2011. MR2744610 (2011m:17058)

[9] V. G. Kac and A. K. Raina, Bombay lectures on highest weight representations of infinitedimensional Lie algebras, Advanced Series in Mathematical Physics, vol. 2, World Scientific Publishing Co., Inc., Teaneck, NJ, 1987. MR1021978 (90k:17013)

[10] Antun Milas, Fusion rings for degenerate minimal models, J. Algebra 254 (2002), no. 2, 300-335, DOI 10.1016/S0021-8693(02)00096-0. MR1933872 (2003k:17037)

[11] James Lepowsky and Haisheng Li, Introduction to vertex operator algebras and their representations, Progress in Mathematics, vol. 227, Birkhäuser Boston, Inc., Boston, MA, 2004. MR2023933(2004k:17050)

[12] Haisheng Li, Determining fusion rules by $A(V)$-modules and bimodules, J. Algebra 212 (1999), no. 2, 515-556, DOI 10.1006/jabr.1998.7655. MR1676853 (2000d:17032)

[13] X. Lin, ADE subalgebras of the triplet vertex algebra $W(p): E_{6}, E_{7}$, preprint.

[14] Alvany Rocha-Caridi and Nolan R. Wallach, Characters of irreducible representations of the Lie algebra of vector fields on the circle, Invent. Math. 72 (1983), no. 1, 57-75, DOI 10.1007/BF01389129. MR696690 (85a:17010)

[15] Weiqiang Wang, Rationality of Virasoro vertex operator algebras, Internat. Math. Res. Notices 7 (1993), 197-211, DOI 10.1155/S1073792893000212. MR.1230296 (94i:17034)

College of Mathematics and Computer Science, Fujian Normal University, Fuzhou, 350108, People's Republic of China

E-mail address: linxianzu@126.com 